\title{
Butelase 1: A Versatile and Multi-purpose Ligase
}

\author{
James P. Tam, Giang K.T. Nguyen, and Xinya Hemu \\ School of Biological Sciences, Nanyang Technological University, 60 Nanyang Drive, 637551, Singapore
}

\section{Introduction}

Proteases are ubiquitous and $>400,000$ putative sequences are found in databases. In contrast, ligases which are peptide-forming enzymes catalyzing the reverse reaction are rare. Thus far only four are identified. Recently, we reported the discovery of butelase 1, a versatile and multi-purpose ligase which is specific for the $C$-terminal Asn/Asp (Asx) ligation.

Butelase 1, isolated from Clitoria Ternatea of the legume family, requires an Asn/Asp (Asx) residue as the recognition site and a sorting signal such as a tripeptide motif Asx-HisVal at the $C$-terminus of a peptide or protein substrate with HisVal dipeptide as a leaving group (Figure 1) [1]. Butelase 1 accepts most amino acids as a nucleophile to form an Asx-Xaa bond without a trace of the sorting signal. Among the known ligases including sortase A, PATG and PCYC1, butelase 1 is the fastest ligase with catalytic efficiencies as high as $1,300,000 \mathrm{M}^{-1} \mathrm{~s}^{-1}$. These favorable properties bode well for applications of butelase 1 for ligation, macrocyclization and labeling of peptides, proteins and live cells. Here, we present our recent work of butelase 1 in peptide macrocyclization using sunflower trypsin inhibitor and histatin as examples.

a



C

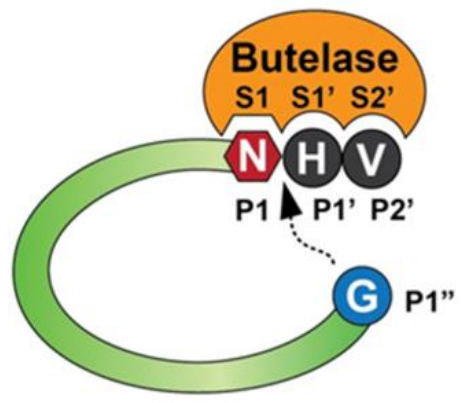

b
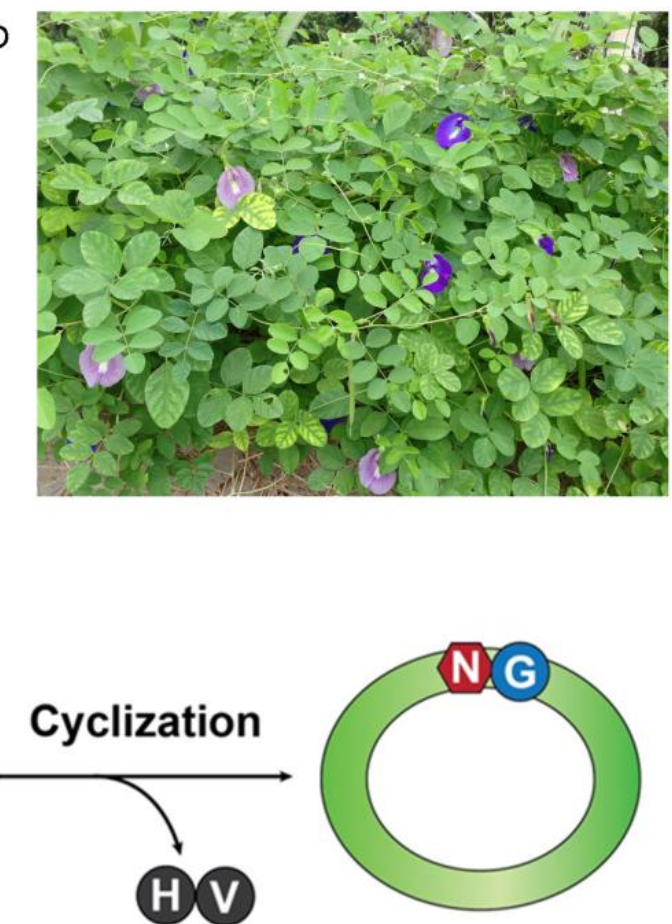

Fig. 1. Schematic illustration of peptide macrocyclization of by butelase. A. Homology modeling of butelase 1, B. Clitoria Ternatea, and C. Macrocyclization mediated by butelase 1 with HV dipeptide as a sorting signal. 


\section{Results and Discussion}

\section{Sunflower Trypsin Inhibitor and its analog}

SFTI-1 is a naturally occurring cyclic peptide from sunflower seeds (Helianthus annuus). It consists of 14 amino acids, stabilized by one disulfide bond and is one of the most potent known inhibitor of trypsin [2]. Figure 2 shows that butelase 1 efficiently cyclized SFTI-1 in $>95 \%$ yield with kinetic parameters of $0.6 \mathrm{~s}^{-1}$ for kcat, $51 \mu \mathrm{M}$ for Km and an overall catalytic efficiency of $11,700 \mathrm{M}^{-1} \mathrm{~s}^{-1}$.

To improve the cyclization efficiency, we synthesized an analog of SFTI-1 with Ile at the P2" position instead of Arg because most native substrates of butelase 1 have a hydrophobic amino acid (Ile/Leu/Val) at this position. The kcat, $\mathrm{Km}$, and catalytic efficiency for SFTI analog were found to be $3.80 \mathrm{~s}^{-1}, 33 \mu \mathrm{M}$, and $115,000 \mathrm{M}^{-1} \mathrm{~s}^{-1}$, respectively. The catalytic efficiency of SFTI analog is approximately 10 times faster than the SFTI-1 (Table 1), confirming the preference for a hydrophobic amino acid of butelase 1 at the $\mathrm{P} 2$ " position.

Table 1. Kinetics parameters of SFTI-1 and SFTI analog.

\begin{tabular}{lcccc}
\hline Peptide & Sequence & $k_{\text {cat }}\left(s^{-1}\right)$ & $k_{M}(\mu M)$ & $k_{\text {cat }} / k_{M}\left(M^{-1} s^{-1}\right)$ \\
\hline SFTI-1 & GRCTKSIPPICFPNHV & 0.6 & 51 & 11,700 \\
SFTI $_{\text {analog }}$ & GICTKSIPPICFPNHV & 3.8 & 33 & 115,000 \\
\hline
\end{tabular}
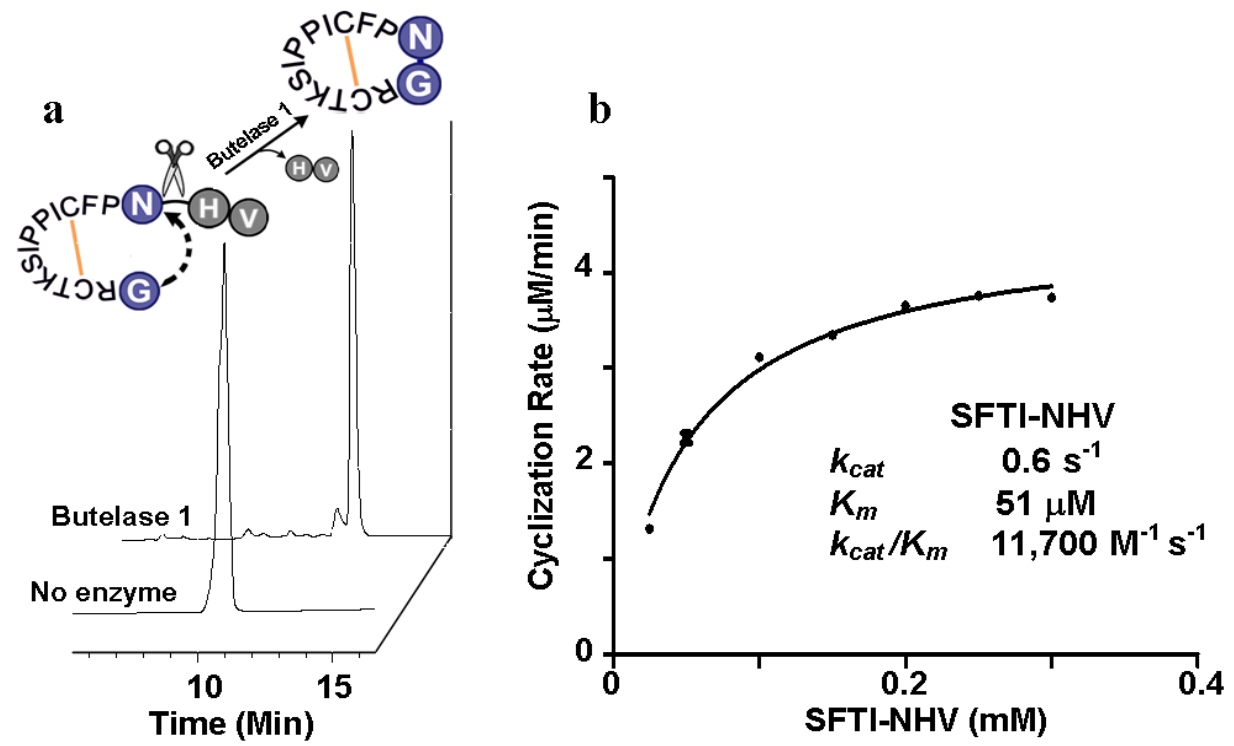

Fig. 2. Butelase mediated cyclization of SFTI-1.

\section{Cyclization of Histatin 1}

Histatin is a naturally occurring peptide antimicrobial found in human saliva. Cyclization of histatin has been shown to improve the activity by a 1000 fold [3]. Figure 2 shows the cyclization of histatin-1 was highly efficient with $>95 \%$ yield in $15 \mathrm{~min}$ in the presence of 0.002 molar equivalent of butelase 1 . For comparison, histatin cyclization by sortase A required 0.33 molar equivalent and $24 \mathrm{~h}$ incubation time to reach $90 \%$ yield. Butelase 1 thus cyclizes the histatin peptide 10,000 times faster than sortase A. 




Fig. 3. Butelase-mediated cyclization of histatin-1. The cyclization reaction was performed at $42{ }^{\circ} \mathrm{C}$ in the presence of $0.1 \mu M$ butelase 1 and $50 \mu M$ peptide substrate. The reaction was monitored by $M S$ over a time course of 15 min. Peptides shown in the MS profiles carried a net charge of +2 .

\section{Conclusion}

Currently, there are two orthogonal ligation approaches without the use of a protection group scheme. The first is the $N$-terminal-specific ligation using a bifunctional $N$-terminal amino acid such as Cys, Ser or Thr [4,5]. This popular approach is well developed in the past two decades and can be performed either chemically or by an intein [6]. The second approach is the $C$-terminal-specific ligation. It requires the use of an enzyme, a ligase, to recognize a specific amino acid at or near the $C$-terminus [7]. This approach is highly underdeveloped because naturally occurring ligases are rare and our discovery of butelase 1 as an Asx-specific ligase provides a promising tool to fill this void. An advantage of butelase is its fast kinetic. It generally completes the cyclization ( 8 to $300+$ aa) within minutes. This characteristic of fast kinetics is important not only because of high yield but also because it minimizes the reverse reaction of random cleavage of Asx residues of the products.

\section{Acknowledgments}

This work was supported in part by the Singapore National Research Foundation grant NRF-CRP8-2011-05.

\section{References}

1. Nguyen, G.K., et al. Nat. Chem. Biol. 10, $732-738$ (2014), http://dx.doi.org/10.1038/nchembio.1586

2. Luckett, S., et al. J. Mol. Biol. 290, 525-533 (1999), http://dx.doi.org/10.1006/imbi.1999.2891

3. Bolscher, J.G., et al. FASEB J. 25, 2650-2658 (2011), http://dx.doi.org/10.1096/fi.11-182212

4. Dawson, P.E., et al. Science 266, 776-779 (1994), http://dx.doi.org/10.1126/science.7973629

5. Liu, C.F., J.P. Tam, J.P. J. Am. Chem. Soc. 116, 4149-4153 (1994), http://dx.doi.org/10.1021/ia00089a001

6. Xu, M.Q., Evans, T.C. Methods 24, 257-277 (2001), http://dx.doi.org/10.1006/meth.2001.1187

7. Mao, H., et al. J. Am. Chem. Soc. 126, 2670-2671 (2004), http://dx.doi.org/10.1021/ja039915e 